\title{
Free Volume Microprobe Studies on Poly(methyl methacrylate)/Poly(vinyl chloride) and Poly(vinyl chloride)/Polystyrene Blends
}

\author{
G.N. Kumaraswamy, C. Ranganathaiah \\ Department of Studies in Physics, University of Mysore, Manasagangotri, Mysore 570 006, India
}

\begin{abstract}
Blends of Poly(methyl methacrylate) (PMMA)/Poly(vinyl chloride) (PVC) and Poly(vinyl chloride) (PVC)/Polystyrene (PS) of different compositions were prepared by solution casting technique. The blends were characterized using Differential Scanning Calorimetry (DSC), Fourier Transform Infrared Spectroscopy (FTIR), and Positron Lifetime Spectroscopy. DSC data were found to be inadequate to describe whether PMMA/PVC blends are miscible or not, possibly because of the small gap in their glass transition temperatures. On the other hand, PVC/PS blends were clearly found to be immiscible by DSC. FTIR results for PMMA/PVC indicate the possible interactions between the carbonyl group of PMMA and $\alpha$-hydrogen of PVC. Free volume data derived from Positron lifetime measurements showed that the PMMA/ PVC blends to be miscible in low PVC concentration domain. For the first time, the authors have evaluated the hydrodynamic interaction parameter $\alpha$, advocated by Wolf and Schnell, Polymer, 42, 8599 (2001), to take into account the friction between the component molecules using the free volume data. This parameter $(\alpha)$ has a high value $(-57)$ at 10 wt $\%$ of PVC, which could be taken to read miscibility for PMMA/PVC blends to be high. In the case of PVC/PS blends, the positron results fully support the DSC data to conclude the blends to be immiscible throughout the range of concentration. As expected, the hydrodynamic interaction parameter $\alpha$ does not show any change throughout the concentration in PVC/PS blends, further supporting the idea that $\alpha$ is another suitable parameter in the miscibility study of polymer blends. POLYM. ENG. SCI., 46:1231-1241, 2006. () 2006 Society of Plastics Engineers
\end{abstract}

\section{INTRODUCTION}

Polymer blending is a widely used technique to improve the physical/mechanical properties of homopolymers [1]. Blending of two polymers may either result in a compatible (miscible) system or incompatible (immiscible) system.

\footnotetext{
Correspondence to: C. Ranganathaiah; e-mail: cr@physics.uni-mysore. ac.in; crang1@rediffmail.com

DOI 10.1002/pen.20566

Published online in Wiley InterScience (www.interscience.wiley. com).

(C) 2006 Society of Plastics Engineers
}

Some level of thermodynamic compatibility between the components is necessary to prevent phase separation during the process resulting in a material of improved properties [2]. A miscible polymer blend means single phase system and the miscibility may be generated mainly in two ways: through the intermolecular specific interactions, such as hydrogen bonding, dipole interactions, etc., and the other is the one involving at least one copolymer where the intramolecular repulsion between the copolymer chains drives the system to miscibility. It is well known that miscibility influences the material properties to a great extent $[2,3]$. Therefore, study of miscibility has received great attention in polymer blend research because of the technological applications of these materials. Besides intermolecular interactions between the component polymer chains, free volume also plays a vital role in deciding the miscibility. Kwak et al. [4] in their study of blends of PVC with BPS-PAr (Bisphynol sulfone-Poly arylate) and TMBPS-PAr (Tetra methyl Bisphynol sulfone-Poly arylate) have reported that despite the presence of same kind of interactions in both the polymer blends, PVC/TMBPS-PAr turns out to be a miscible system whereas PVC/BPS-PAr results in an immiscible system. In another case, PS (Polystyrene)/PC (Bisphenol-A Polycarbonate) and PS (Polystyrene)/TMPC (Tetra methyl Bisphenol-A Polycarbonate) blends were studied by Liu et al. [5]; the latter system was found to be miscible, whereas the former system resulted in an immiscible blend. In both of the above cases, the observed miscibility was interpreted because of the additional free volume created by the presence of four methyl groups on the side chains of TMBPSPAr and TMPC. These studies clearly suggest that in the absence of strong specific interactions between the chains of blend, it is expected that free volume of the homopolymers play an important role in generating the miscibility.

It is known that hydrodynamic interactions exit between the constituents of a polymer blends and these interactions influence the viscometric behavior of the system. Discussions on hydrodynamic interactions in polymer solutions (polymer/solvent system) can be found in detail in the form of well-known models such as Kargin-Slonimsky-Rouse [6] and Kirkwood-Riseman-Zimm [7]. Recently, Wolf and (W)WILEY 
Schnell [8] have discussed the effect of hydrodynamic interactions in polymer solutions through their model and calculated a parameter called hydrodynamic interaction parameter $\alpha$ using viscometric data. This parameter is found to be especially useful in providing information on the mixing level in the blend system. They also proposed that this theory could as well be used to polymer/polymer blends in solid phase. In the present study, we have made an attempt to generalize Wolf and Schnell theory to polymer/polymer systems in solid phase and evaluated the hydrodynamic interaction parameter using free volume data for the first time instead of viscosity. An attempt is made to describe the miscibility of the system based on behavior of $\alpha$. In the absence of strong intermolecular interactions, it seems that the parameter $\alpha$ could be used to judge the miscibility of the system better.

A survey reveals that Poly(methyl methacrylate) (PMMA)/Poly(vinyl chloride) (PVC) blends have been extensively studied specially on the miscibility of this system using various experimental techniques. We observe that there are few contradictory reports regarding the miscibility range in this blend. Schurer et al. [9] who studied this system using Differential Scanning Calorimetry (DSC), DMA, and turbidity measurements concluded the system as miscible in low PMMA concentrations (less than $40 \mathrm{wt} \%$ ). Later, Vanderschueren et al. [10] studied the same system using Thermally Stimulated Depolarization Currents method and concluded that the miscibility range of this system was less than $10 \mathrm{wt} \%$ of PMMA. Interestingly, few other groups have reported this system to be miscible throughout the composition range [11, 12]. At the same time, there are opinions that the determination of miscibility in polymer blends depends on the method adopted and the temperature range of investigations. Further, there are several examples in literature about the controversy around the level of miscibility for a given polymer pair according to DSC, optical, Mechanical, Dielectric, NMR, Viscometric measurements [9, 13-15]. In this study, we report the use of a novel technique such as Positron Lifetime Technique (PLT) to study the miscibility of the polymer blends, although it is not for the first time that a study provides molecular level picture of the blend system.

PLT is a versatile technique used for decades in the study of free volume properties of polymers and recently of polymer blends [16-19]. It measures the free volume size and its content in the system. Through several of these studies, it has been well established that free volume in polymers influence the macroscopic properties of the polymers and blends [20-24]. Therefore, a measure of free volume certainly provides an insight into the macroscopic behavior of the system. The following paragraph briefly outlines the basis of PLT.

A positron from a radioactive source (commonly $\mathrm{Na}-22$ ) when injected into a molecular medium, such as polymer, interacts with the medium and loses its kinetic energy in a very short time (around $1 \mathrm{ps}$ ), and reaches thermal energy. The thermalized positron may pick up an electron from the medium and annihilate as a free positron, or get trapped into defects present in the crystalline, crystalline-amorphous interface regions of the system and then annihilate, or form a bound state with an electron of the medium $\left(\mathrm{e}^{+} \mathrm{e}^{-}\right)$called the Positronium (Ps) atom. Positronium exits in two allowed spin states: para-Positronium (p-Ps), if the spins of $\mathrm{e}^{+}$and $\mathrm{e}^{-}$are aligned antiparallel and annihilates into two gamma photons with a lifetime of $0.125 \mathrm{~ns}$; the ortho-Positronium (o-Ps), in which spins are parallel and annihilates with a lifetime of $140 \mathrm{~ns}$ in free space. However, in molecular media like polymers, the positron of o-Ps picks up an electron from the surrounding medium and annihilates through a fast channel called pick-off annihilation and its lifetime gets shortened to few nanoseconds. The fact that o-Ps preferentially localizes in the free volume holes of polymers [25] from which it annihilates, makes it the microprobe of free volume holes since its lifetime and intensity are related to free volume size and its content. As such these measurements have been widely used over the last few decades in the study of microstructural behavior of polymers and recently in blends. It is a practice to correlate the free volume hole size and its concentration to the viscoelastic properties of the system under investigation. Therefore, the study of free volume in polymer blends provides an understanding of the molecular level mixing in blends.

Motivated by the observations made above, we have selected two blends for our study. One is the PMMA (Polymethyl methacrylate)/PVC (Polyvinyl chloride), where one may expect intermolecular interactions between the carbonyl group of PMMA and $\alpha$-hydrogen of PVC. However, this interaction is rather weak and leaves the blend as partially miscible. The other system selected is PVC (Polyvinyl chloride)/PS (Polystyrene) blend in which no intermolecular interactions are possible. Therefore, the focus of this study is to understand what information can be unearthed from the free volume measurement in such systems. We have carried out free volume measurements for different composition of the above mentioned blends. Besides PLT, we have also employed DSC and Fourier Transform Infrared Spectroscopy (FTIR) as complementary techniques to determine the blend miscibility.

\section{EXPERIMENTAL}

\section{Blends Preparation}

Samples of PMMA, PVC, and PS having densities 1.2 $\mathrm{g} / \mathrm{cc}, 1.34 \mathrm{~g} / \mathrm{cc}$, and $1.04 \mathrm{~g} / \mathrm{cc}$ with weight average molecular weights of $15,000,43,000$, and 1,90,000 g/mol, respectively, were procured from M/s Sigma-Aldrich Chemicals Limited. Using these samples in as received condition the blends were prepared by the conventional solution casting method. The weighed fractions of PMMA and PVC were dissolved in Tetrahydrofuran at $60^{\circ} \mathrm{C}$ at different proportions starting from 90/10,80/20,70/30, etc., to $10 / 90$ and the solution was cast on a clean and flat glass plate. After 
allowing the solvent to evaporate at room temperature, the films were lifted from the glass plate. The neat films so obtained were of approximately of $1 \mathrm{~mm}$ thickness. Similarly, different proportions of PVC and PS were dissolved in methyl ethyl ketone and blends of different weight concentrations were prepared by casting the solution on to a clean glass plate. The PMMA/PVC blend samples were observed to be optically transparent at all concentrations but PVC/PS samples were opaque throughout the concentration range. The blend samples so prepared were vacuum dried at $85^{\circ} \mathrm{C}$ for about $10 \mathrm{~h}$ to remove the residual solvent. All the samples were stored in a desiccator before the actual use in experiments.

\section{Differential Scanning Calorimetric Measurements}

The glass transition temperatures $\left(T_{\mathrm{g}}\right)$ of the pure polymers and their blends were measured using a Universal V3.0G TA instrument DSC 2010 connected to liquid nitrogen cooling accessory with a nitrogen purge. Each experiment began with cooling from room temperature to $0^{\circ} \mathrm{C}$ at the rate of $10^{\circ} \mathrm{C} / \mathrm{min}$. Then the samples were allowed to equilibrate for $10 \mathrm{~min}$ at $0^{\circ} \mathrm{C}$ before starting the measurements. The $T_{\mathrm{g}}$ of the pure polymers and the selected blends of 70/30, 50/50, 30/70 compositions were measured. Samples of $10 \mathrm{mg}$ weight were used with a heating rate of $10^{\circ} \mathrm{C} / \mathrm{min}$ starting from $0^{\circ} \mathrm{C}$ to $200^{\circ} \mathrm{C}$.

\section{FTIR Measurements}

FTIR spectra were recorded on a JASCO FT/IR-460 Plus instrument in the range $4000-400 \mathrm{~cm}^{-1}$ at room temperature. The samples were prepared by mixing the fine powder of the blend sample with $\mathrm{KBr}$ powder for the FTIR measurements.

\section{Positron Annihilation Lifetime Measurements}

Positron annihilation lifetime spectra were recorded for the pure polymers and their blends using Positron Lifetime Spectrometer. The spectrometer consists of a fast-fast coincidence system with $\mathrm{BaF}_{2}$ scintillators coupled to photomultiplier tubes type XP2020/Q with quartz window as detectors. The $\mathrm{BaF}_{2}$ scintillators were conical shaped to achieve better time resolution. The two identical pieces of the sample were placed on either side of a $17 \mu \mathrm{Ci}{ }^{22} \mathrm{Na}$ positron source, deposited on a pure Kapton foil of $0.0127 \mathrm{~mm}$ thickness. This sample-source sandwich was placed between the two detectors of the spectrometer to acquire lifetime spectrum. The ${ }^{60} \mathrm{Co}$ source was used to acquire the prompt time spectrum, which gave $180 \mathrm{ps}$ as the resolution of the spectrometer. However, to reduce the acquisition time and increase the count rate, the spectrometer was operated at 220 ps. All lifetime measurements were performed at room temperature with more than a million counts under each spectrum recorded in a time of 1 to $2 \mathrm{~h}$. Source correction term and resolution functions were estimated from the lifetime of well-annealed aluminum using the program RESO-
LUTION [26]. Since single Gaussian resolution function did not yield convergence, the resolution function was resolved further into three Gaussian components, which produced quick and good convergence. The net resolution function, however, for this remained at $220 \mathrm{ps}$. The compliancy of the resolution function was tested with wellcharacterized polymer samples like PC and PTFE and the results obtained agreed very well with literature reported values. Therefore, three Gaussian resolution functions were used in the present analysis of positron lifetime spectra in all the blends and pure polymer samples. All spectra were analyzed into three lifetime components with the help of the computer program PATFIT-88 [26] with proper source and background corrections.

\section{RESULTS AND DISCUSSION}

\section{$\mathrm{T}_{g}$ Measurements}

Determination of $T_{\mathrm{g}}$ is a widely used method to study the miscibility in polymer blends. $T_{\mathrm{g}}$ is the characteristic temperature of any polymer at which the polymeric system changes from hard and glassy state to flexible rubbery state. This happens because of the segmental motion of the polymeric chains. If a blend turns out be miscible or exhibits homogeneous phase, chains of both the polymers relax cooperatively resulting in single $T_{\mathrm{g}}$. On the other hand, if the blend is immiscible, chains of the component polymers relax independently at different temperatures giving two $T_{\mathrm{g}} \mathrm{s}$. In the present case, we have measured the $T_{\mathrm{g}} \mathrm{s}$ of homopolymers and their blends of 70/30, 50/50, and 30/70 compositions. In Fig. 1a, the DSC thermograms of homopolymers PMMA and PVC and their blends of 70/30, 50/50, and 30/70 compositions are given. From figure, we notice that PMMA is exhibiting a broad glass transition that starts around $84^{\circ} \mathrm{C}$ and extends up to $98^{\circ} \mathrm{C}$. The component polymer PVC shows its $T_{\mathrm{g}}$ at $80^{\circ} \mathrm{C}$. In the same figure, we have shown the thermograms for the blends of PMMA/PVC 70/30, 50/50, and 30/70. These exhibit single glass transitions at around $84^{\circ} \mathrm{C}$. It should be noticed that the component polymers of PMMA/PVC blends have $T_{\mathrm{g}}$ values which are very close, the blends exhibiting single glass transition like seems to mislead with regard to miscibility. Thereby, the observed single glass transition cannot be considered as indication of miscibility in PMMA/PVC system. Therefore, for the present system, it could be inferred that determination of $T_{\mathrm{g}}$ alone using DSC seems to be not sufficient to reveal whether the blends are miscible or immiscible.

DSC scans of PVC/PS system are presented in Fig. 1b. From this figure, we can clearly observe that $T_{\mathrm{g}}$ of $\mathrm{PVC}$ is $80^{\circ} \mathrm{C}$ and that of PS is $98^{\circ} \mathrm{C}$. The PVC/PS blends of $70 / 30$, $50 / 50$, and 30/70 compositions exhibit clear two glass transitions, each one corresponding to their homopolymers glass transitions. Therefore, on the basis of the DSC results, it can be concluded positively that PVC/PS blends are immiscible systems. 


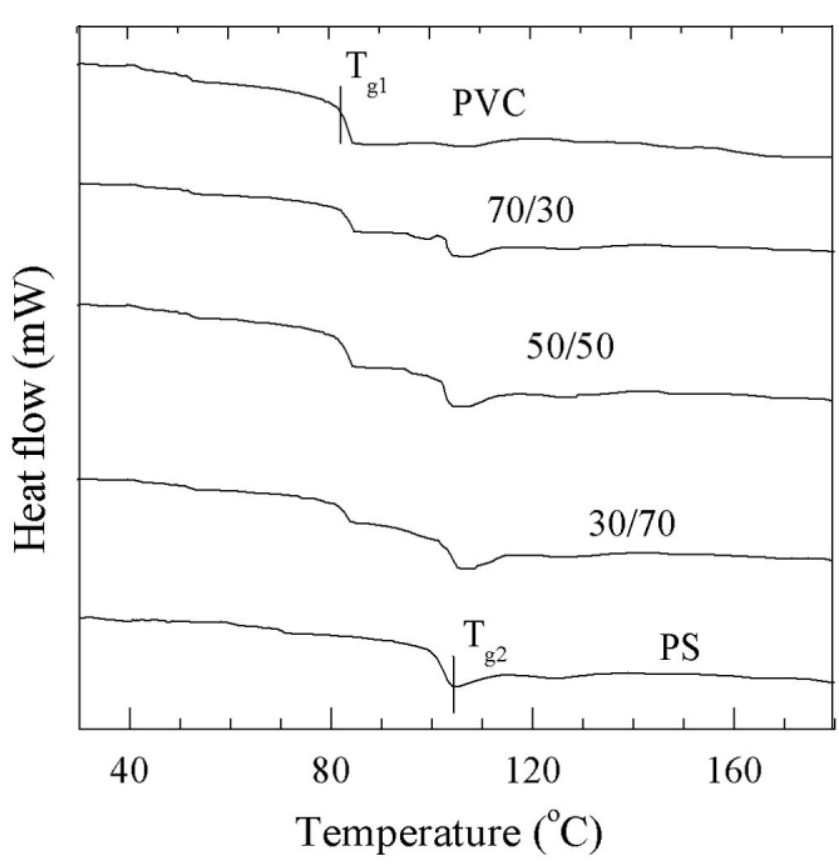

(a)

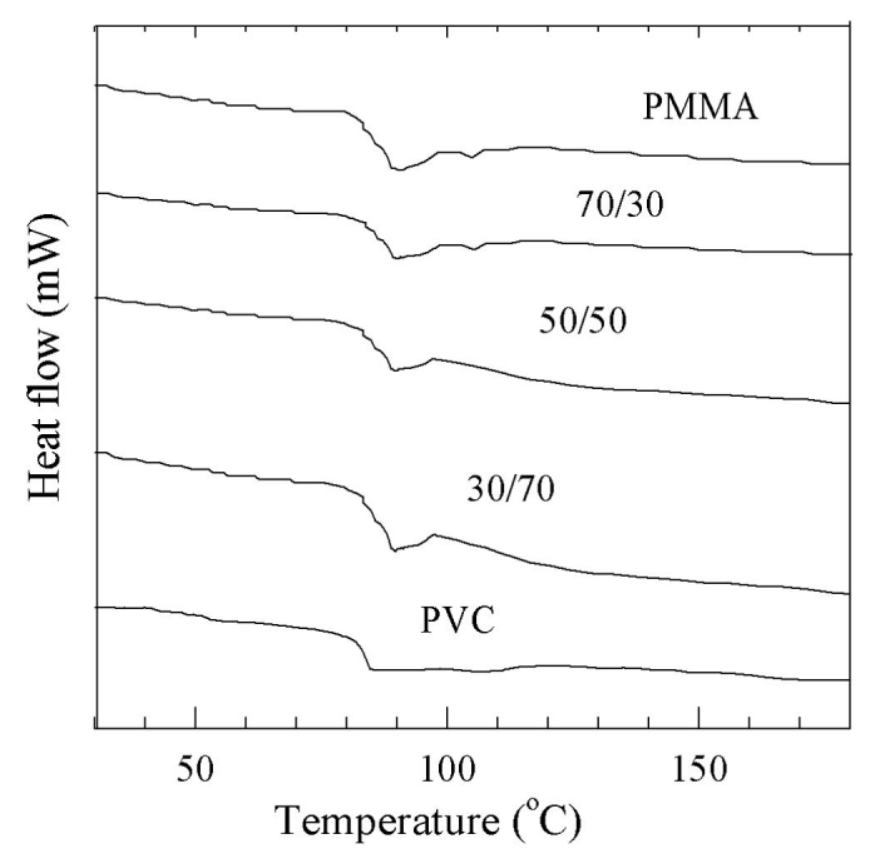

(b)

FIG. 1. (a) DSC thermograms of PMMA, PVC, and their blends of 70/30, 50/50, and 30/70. (b) DSC thermograms of PVC, PS, and their blends of 70/30, 50/50, and 30/70.

\section{Infrared Spectroscopy Results}

Normally, infrared measurements in blends are carried out to explore the possible interactions between the blend components. In the present study, we have performed FTIR measurements with the same aim to ascertain possible interactions between the PMMA and PVC chains. Figure 2 displays infrared spectra of PMMA and selected blends of PMMA/PVC 70/30 and 50/50 blends. The carbonyl group

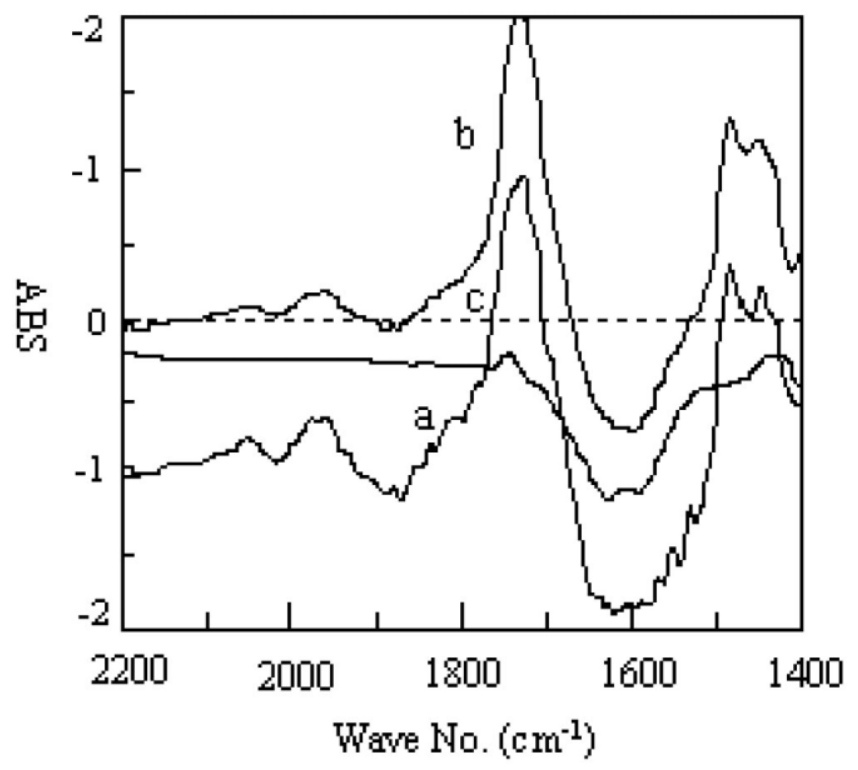

FIG. 2. FTIR spectra of (a) PMMA, and PMMA/PVC blends of (b) $70 / 30$, (c) $50 / 50$.

of PMMA being polar has the possibility of involving in dipolar or hydrogen type of interactions with the other polymers. The $\alpha$-hydrogen of PVC is also slightly polar. Therefore, we expect an interaction between carbonyl group of PMMA and $\alpha$-hydrogen of PVC. From Fig. 2, we can clearly observe a shift in carbonyl group band from 1742 $\mathrm{cm}^{-1}$ in pure PMMA to $1730 \mathrm{~cm}^{-1}$ in its blends. The shift in carbonyl absorption band frequency to lower side is considered as an indicator of negative heat of mixing [27], which results from the attractive interactions between the carbonyl group of PMMA and $\alpha$-hydrogen of PVC [28, 29]. As mentioned earlier, miscibility results from the attractive intermolecular interactions between the blend components. Therefore, the observed shift in the absorption band explained above is attributable to miscibility of PMMA/PVC blends. For both the blends (70/30 and 50/50), the shift was same, suggesting the strength of the interaction is of the same order in both these concentrations.

\section{Positron Lifetime Results: Free Volume Data}

All the measured lifetime spectra were resolved into three lifetime components $\tau_{1}, \tau_{2}$, and $\tau_{3}$ with corresponding intensities $\mathrm{I}_{1}, \mathrm{I}_{2}$, and $\mathrm{I}_{3}$ since this analysis gave better $\chi^{2}$ values and standard deviations than the two and four component analysis. Hence three component analysis results are described here. The attribution of these lifetime components is generally as follows [25]. The shortest lifetime component $\tau_{1}$ with intensity $\mathrm{I}_{1}$ is attributed to the contributions from p-Ps and free positron annihilations. The intermediate lifetime component $\tau_{2}$ with intensity $\mathrm{I}_{2}$ is mainly due to annihilation of positrons trapped at the defects present in the crystalline regions or trapped at the crystalline-amorphous interface regions. The longest-lived component $\tau_{3}$ with in- 
tensity $\mathrm{I}_{3}$ is due to pick-off annihilation of the o-Ps from the free volume sites present mainly in the amorphous regions of the polymer matrix [30]. Of these three lifetime components, it is the o-Ps lifetime $\tau_{3}$ that is related to the free volume hole size by a simple relation given by Nakanishi et al. [31], which was developed on the basis of theoretical models originally proposed by Tao [32] for molecular liquids and later by Eldrup et al. [33] for molecular solids. In this model, Positronium is assumed to be localized in a spherical potential well having an infinite potential barrier of radius $R_{o}$ with an electron layer in the region $R<r<R_{o}$. The relation between $\tau_{3}$ and free volume cavity $R$ from which the o-Ps annihilates is given as

$$
\left(\tau_{3}\right)^{-1}=2\left[1-\frac{R}{R_{0}}+\frac{1}{2 \pi} \sin \left(\frac{2 \pi \mathrm{R}}{\mathrm{R}_{0}}\right)\right] \mathrm{ns}^{-1}
$$

where $\mathrm{R}_{\mathrm{o}}=\mathrm{R}+\delta \mathrm{R}$ and $\delta \mathrm{R}$ is an adjustable parameter. By fitting $E q .1$ with $\tau_{3}$ values for known hole sizes in porous materials like zeolites, a value of $\delta \mathrm{R}=0.166 \mathrm{~nm}$ was obtained. It has been verified that the same value of $\delta \mathrm{R}$ is valid for the pure polymers and their blends investigated in this work. With this value of $\delta \mathrm{R}$, the free volume radius $\mathrm{R}$ has been evaluated using $E q .1$ and the average size of free volume holes $V_{f}$ is calculated as $V_{f}=(4 / 3) \pi R^{3}$. The relative fractional free volume or the free volume content $\left(\mathrm{F}_{\mathrm{vR}}\right)$ of the system can then be estimated as

$$
\mathrm{F}_{\mathrm{vR}}=\mathrm{V}_{\mathrm{f}} \mathrm{I}_{3} .
$$

According to free volume theory of Ps formation, $\tau_{3}$ correspond to the volume of cavities present in the system and $\mathrm{F}_{\mathrm{vR}}$ is the relative measure of free volume fraction of the system. Therefore, free volume hole size $\mathrm{V}_{\mathrm{f}}$ and the relative fractional free volume $\mathrm{F}_{\mathrm{vR}}$ are the parameters that are used to characterize the polymer blends in the present work.

Free volume in polymeric systems evolves because of the empty spaces between and along the polymer chains. Therefore, the free volume hole size and the free volume fraction depend on the chain structure, spacing, and orientations. Polymeric systems having ordered arrangement of polymeric chains with close packing give rise to smaller free volume. When two polymers are blended and blending results in some specific interactions between the chains of the component polymers, the orientation of chains in a particular manner results to close packing. If this occurs, it results in reduced free volume of the system. This reduction can be observed by comparing the free volume of the blend with the free volume calculated according to linear additivity relation. Literature reveals that free volume hole size, $V_{f}$, and relative fractional free volume $\mathrm{F}_{\mathrm{vR}}$ behave differently in miscible and immiscible blends. The miscible blends show negative deviation in $\mathrm{F}_{\mathrm{vR}}$ from the well-known linear additivity relation given as [34],

$$
\mathrm{F}_{\mathrm{vR}}=\mathrm{F}_{\mathrm{vR} 1} \mathrm{~W}_{1}+\mathrm{F}_{\mathrm{vR} 2} \mathrm{~W}_{2}
$$

where $\mathrm{F}_{\mathrm{vR}}$ is the relative fractional free volume of the blend, $\mathrm{F}_{\mathrm{vR} 1}, \mathrm{~F}_{\mathrm{vR} 2}, \mathrm{w}_{1}$ and $\mathrm{w}_{2}$ are the relative fractional free volumes and corresponding weight fractions of the blend constituents 1 and 2. In case of immiscible blends, $F_{\mathrm{vR}}$ show mostly positive deviation or agreed with linear additivity relation [35] but so far no immiscible blends have been reported to exhibit negative deviation of these parameters.

It is known that only the occupied volume of the blend components is additive, but the free volume appears to be a nonadditive quantity. Therefore, based on the theory of $\mathrm{Wu}$ [36], Liu et al. [5] have given a relation to estimate the relative free volume fraction in a blend as,

$$
\mathrm{F}_{\mathrm{vR}}=\mathrm{F}_{\mathrm{vR} 1} \phi_{1}+\mathrm{F}_{\mathrm{vR} 2} \phi_{2}+\beta \mathrm{F}_{\mathrm{vR} 1} \mathrm{~F}_{\mathrm{vR} 2} \phi_{1} \phi_{2}
$$

where $\mathrm{F}_{\mathrm{vR}}$ stand for the usual notation and $\phi_{1}, \phi_{2}$ corresponds to the volume fractions of the constituent polymers 1 and 2 , respectively. In the above equation, $\beta$ could be inferred as free volume interaction parameter since it represents the amount of deviation from the additivity rule. From the above relation, using the relative fractional free volumes of the blends and that of the homopolymers one can evaluate the parameter $\beta$ at different compositions of the blend. This $\beta$ has been observed to be negative [5] in case of miscible blends and positive and zero for immiscible blends [19].

PMMA/PVC Blends. The variation of o-Ps lifetime $\tau_{3}$ and the free volume hole size $V_{f}$ as function of blend composition is presented in Fig. 3a. From this we notice the hole size in PMMA is about $115.6 \AA^{3}$ and in PVC much smaller in size of $72 \AA^{3}$. Therefore, it is expected that the hole size shall decrease as the PVC weight fraction in the blend increases. Of course, we do observe the same trend, but the variation does not follow the simple linear additivity rule (Eq. 3), instead it exhibits negative deviation up to 50 wt $\%$ of PVC and positive deviation beyond $50 \mathrm{wt} \%$. The negative deviation of $\mathrm{V}_{\mathrm{f}}$ up to $50 \mathrm{wt} \%$ could be inferred because of arrangement of the polymeric chains in compact form, which suggests specific interactions between PMMA and PVC chains. Beyond $50 \mathrm{wt} \%$ of PVC, the $\mathrm{V}_{\mathrm{f}}$ shows positive deviation, suggesting the evolution of additional free volume because of phase separation. Therefore, changes in $\mathrm{V}_{\mathrm{f}}$ for this system of blends suggest that molecular packing and the level of mixing is good in lower PVC concentration and it decreases with the increase in PVC concentration. Figure $3 \mathrm{~b}$ is a plot of variation of relative fractional free volume as function of blend composition. It is clear that it exhibit negative deviation throughout the composition range, indicating reduced free volume fraction throughout the range of composition. The relative free volume number density $I_{3}$ also shows negative deviation, which is exactly similar to that we observe for $F_{v R}$ (Fig. 3b). Normally, free volume size $\left(\mathrm{V}_{\mathrm{f}}\right)$ and relative fractional free volume $\left(\mathrm{F}_{\mathrm{VR}}\right)$ are considered important parameters rather than $\mathrm{I}_{3}$ in Positron measurements, because $\mathrm{I}_{3}$ depends on 


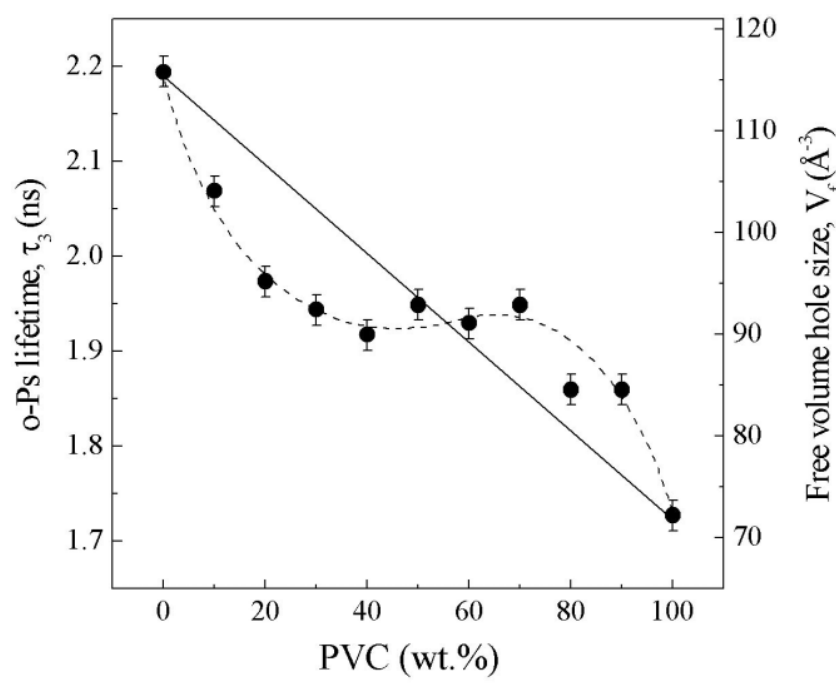

(a)

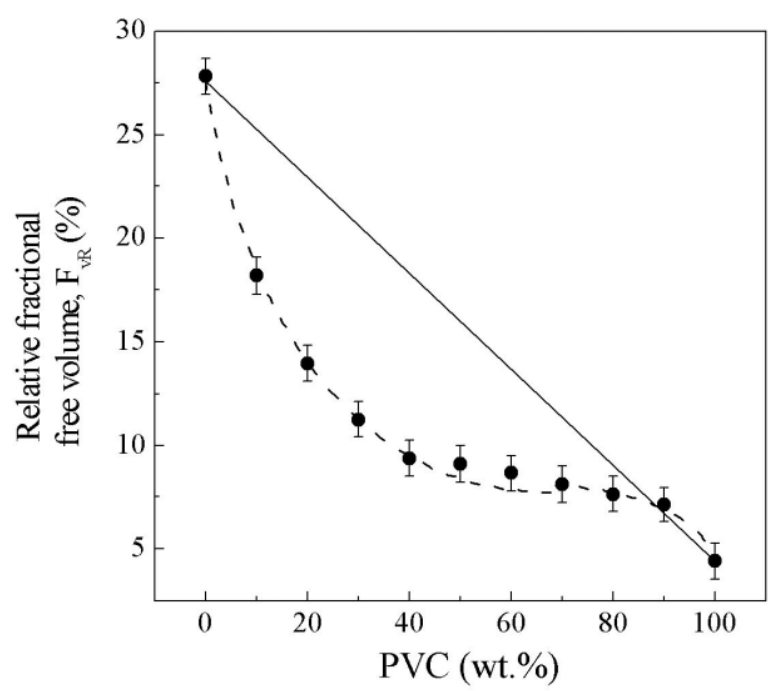

(b)

FIG. 3. (a) Plot of free volume hole size, $V_{f}$, as function of PMMA concentration in the blend. The solid line represents the linear additive relation and dotted line is to guide the eye. (b) Plot of relative fractional free volume, $\mathrm{F}_{\mathrm{vR}}$, as function of PMMA concentration in the blend. The solid line represents the linear additive relation and dotted line is to guide the eye.

several factors [37-39] and it is difficult to place emphasis on this parameter. The combination of $\mathrm{V}_{\mathrm{f}}$ and $\mathrm{F}_{\mathrm{vR}}$ results could suggest that the PMMA/PVC blends are partially miscible and can fix the miscibility in the concentration range up to $50 \mathrm{wt} \%$ of PVC.

The calculated $\beta$ values from Eq. 4 are plotted in Fig. 4. From the figure we observe that $\beta$ attains maximum negative value of -0.74 for $10 \mathrm{wt} \%$ of PVC and it decreases with increase in PVC content of the system. The negative value of $\beta$ shows decrease in free volume of the blend from additivity rule $(E q .4)$, an indication of the miscibility. The behavior of $\beta$ suggests that the miscibility is high in the lower PVC concentrations $(\beta=-0.74)$ and it decreases with increase in PVC content in the blend. The parameter $\beta$,

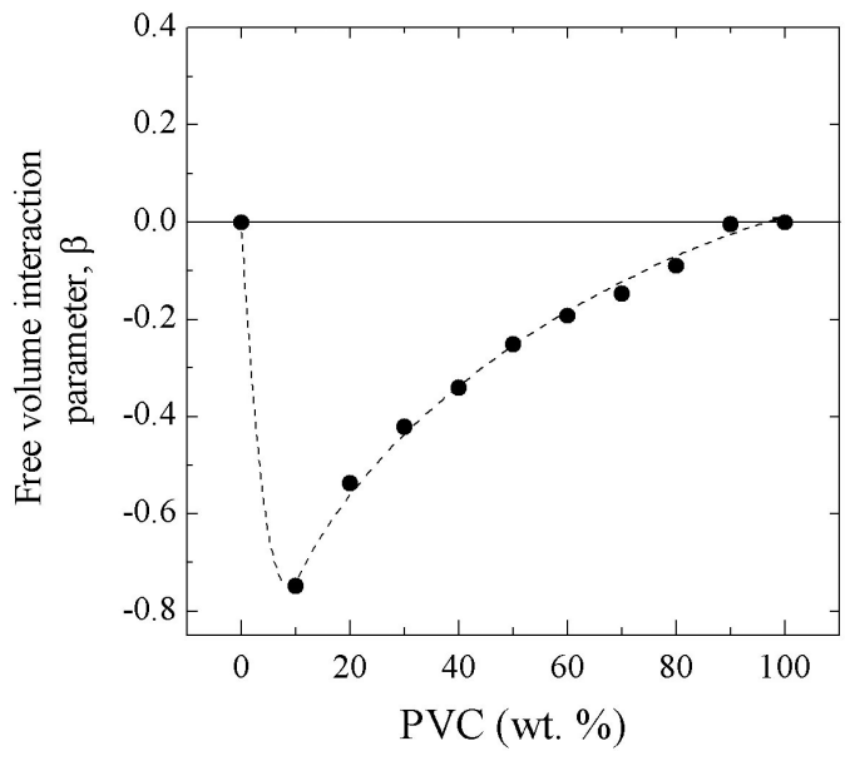

FIG. 4. Plot of the free volume interaction parameter $\beta$ as function of PMMA concentration in the blend. The dotted line is to guide eye.

though interpreted vaguely as free volume interaction parameter, is difficult to explain the meaning of this. It could simply mean the measure of geometrical change as for as free volume evolution is concerned.

As mentioned in the introduction, a model description of the viscosity of polymer/solvent system as pursued by Wolf and Schnell [8], considers the following concepts. The dissipation of energy takes place at the molecular interfaces, the friction between the components varies with composition because of change in flow mechanism (drainage of molecular coils), and the coil volume depends on the concentration. To generalize their approach, Wolf and Schnell extended these to polymer solutions and proposed that experiments will be needed to test whether this holds good for polymer blends in the glassy solid phase. This approach introduces two parameters, namely, $\gamma$, the geometric factor, and $\alpha$, the hydrodynamic interaction parameter. $\gamma$ measures the ratio of surface/volume fractions of the components whereas $\alpha$ is clearly related to the thermodynamics of mixing.

We have made an attempt to make use of this theory and evaluate these parameters using free volume data since viscosity and free volume are inversely related [40]. To this end, we suitably modified Wolf and Schnell relations incorporating free volume parameters. The following relation was used to evaluate $\alpha$ :

$$
\begin{aligned}
& \Delta \mathrm{F}_{\mathrm{vR}}= \\
& \left\{\delta\left[\gamma\left(1+\gamma \phi_{2}\right)^{2}-\phi_{1}(1+\gamma)\right]+2 \alpha(1+\gamma)^{2} \phi_{2}^{2}+e \frac{1}{F_{\mathrm{VR}}} \rho \phi_{1}\right\}^{-1} \\
& \frac{\left(1+\gamma \phi_{2}\right)^{3}}{\phi_{1} \phi_{2}}
\end{aligned}
$$

the left hand side quantity $\Delta \mathrm{F}_{\mathrm{vR}}$ in $E q .5$ is calculated as, 


$$
\Delta F_{\mathrm{vR}}=\left[\frac{1}{F_{\mathrm{vR}}}-\frac{\phi_{1}}{F_{\mathrm{vR} 1}}-\frac{\phi_{2}}{\mathrm{~F}_{\mathrm{vR} 2}}\right]^{-1}
$$

in which the right hand side parameters are known from positron data.

$\delta$ in Eq. 5 is defined as $=\frac{1}{F_{\mathrm{vR} 2}}-\frac{1}{F_{\mathrm{vR} 1}}$, which is the difference in the reciprocal of the free volumes of the pure polymers. In the above equations, $\alpha$ is the hydrodynamic interaction parameter, $\rho$ is the density of the blend, $\varphi_{1}$ and $\varphi_{2}$ are volume fractions of the blend constituents. It has been observed that $\alpha$ attains large negative values in case of miscible systems indicative of good thermodynamic mixing and it decreases and approaches zero with decrease in miscibility level or increase with positive values in phase separated systems.

The geometry factor $\gamma$ in $E q .5$ is evaluated from the following relation [41], since we know $\varphi_{1}, \varphi_{2}, \mathrm{~F}_{\mathrm{vR}}, \mathrm{F}_{\mathrm{vR} 1}$, and $\mathrm{F}_{\mathrm{vR} 2}$.

$$
F_{\mathrm{vR}}=\left[\frac{\phi_{1}}{F_{\mathrm{vR} 1}}+\frac{\phi_{2}}{F_{\mathrm{vR} 2}}+\delta\left(\frac{\gamma \phi_{1} \phi_{2}}{1+\gamma \phi_{2}}\right)\right]^{-1}
$$

$\gamma$ the geometric factor was considered to be constant in the original theory. It is expressed as

$$
\gamma=\frac{N_{2}}{N_{1}}-1
$$

where $\mathrm{N}_{\mathrm{i}}$ is the ratio of surface fraction to volume fraction of component polymers 1 and 2 . This can be determined by using Bondi's group contribution method [42]. For the present system of PMMA/PVC, it turns out to be -0.12 . We have calculated the $\gamma$ value from Eq. 7 by replacing molar surface and molar volume by free volume surface area, $\mathrm{S}$, and relative fractional free volume, $\mathrm{F}_{\mathrm{vR}}$. The free volume surface area, $\mathrm{S}$, was calculated using the empirical relation $\mathrm{I}_{3}=3.0+0.033 \mathrm{~S}$ [43], where $\mathrm{I}_{3}$ is the o-Ps intensity. The value of $\gamma$ so calculated turns out to be -0.15 . This is in very good agreement with the $\gamma$ value calculated from Bond's method. Geometric factor $\gamma$ depends on the molecular surfaces and volumes in the system. Blend composition that influences the phase morphology of the system also influences the molecular surfaces and volumes in the system. Therefore, it is obvious to expect the variation of $\gamma$ with respect to blend composition and this is quite evident from Eq. 6. Using this equation, $\gamma$ values have been calculated at different compositions of the blend and the results are presented in Fig. 5a. Since $\gamma$ variation does not exhibit any systematic changes (it oscillates between positive and negative values), any meaningful conclusion can not be drawn.

Further making use of $\gamma$ values, the hydrodynamic interaction parameter $\alpha$ was evaluated. The hydrodynamic interaction parameter $\alpha$ by definition quantifies the deviation of friction between component 1 and 2 , especially unlike components and measures the excess friction developed in the system due to the interactions between the constituent

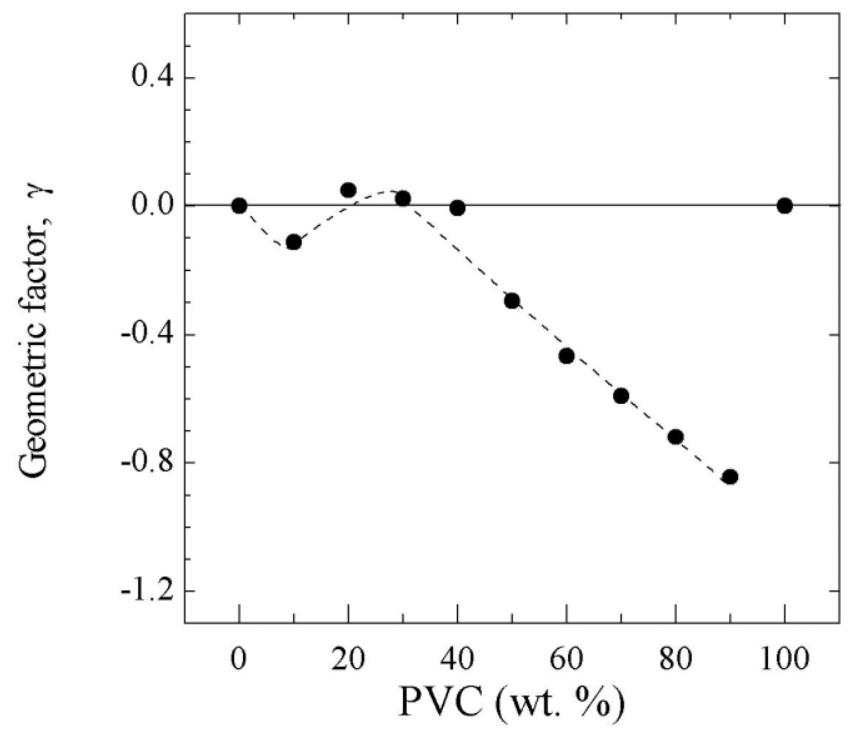

(a)

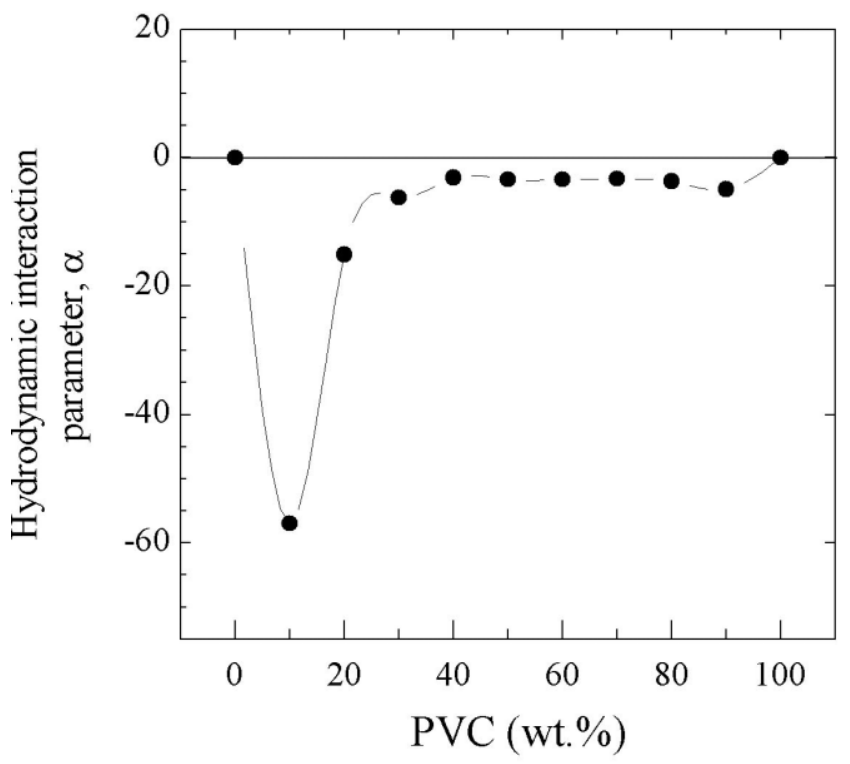

(b)

FIG. 5. (a) Plot of the geometrical parameter $\gamma$ as function of PMMA concentration in the blend. The solid line corresponds to zero and dotted is to guide eye. (b) Plot of hydrodynamic interaction parameter $\alpha$ as function of PMMA concentration in the blend. The solid line corresponds to zero and dotted is to guide eye.

polymer chains. In case of miscible blends where the chains of polymer 1 are evenly distributed in polymer 2 , the sites of interactions between the constituent polymer chains is maximum, thus generating high friction in the system. In such circumstances, $\alpha$ attains large negative values. In case the blend is immiscible, then one polymer forms its own domains in the system, resulting in reduced possible contacts between chains of Polymer 1 and 2 to a great extent. This reduces the friction between the chains of constituent polymers. Such systems produce small $\alpha$ values that are almost equal to zero hence the system becomes immiscible. 
The values of $\alpha$ at different weight composition of PVC in the blend are presented in Fig. 5b. It is evident from the figure that $\alpha$ is maximum (-57) at $10 \mathrm{wt} \%$ of PVC and decreases rapidly with the increase of PVC concentration in the blend and approaches zero in high PVC concentrations. In the light of the above description, the larger value of $\alpha$ at lower PVC concentration (10 $\mathrm{wt} \%$ ) can be considered as due to the good molecular level mixing of the constituent polymers of the blend. The increase in PVC concentration decreases this level of mixing and this is evidenced by reducing in $\alpha$ values.

Based on the $\mathrm{F}_{\mathrm{vR}}$ data obtained from positron measurements, the blends were found to be miscible in the low PVC concentration range, which could go up to $50 \mathrm{wt} \%$ of PVC. This was also supported by the free volume interaction parameter, $\beta$, which showed maximum negative value in low PVC concentration. However, behavior of $\alpha$ the hydrodynamic interaction parameter clearly suggests the miscibility level is maximum at $10 \mathrm{wt} \%$ of PVC. As outlined in the introduction, miscibility studies in PMMA/PVC blends have been extensively conducted using various techniques $[9-12,44,45]$ but no report of the free volume measurements. Most of these studies have reported the system to be miscible only in lower PMMA concentration. Although few have reported it to be miscible throughout the composition range, none of these studies indicated the miscibility to lie in the low PVC concentrations. Fekete et al. [28] proposed that the interactions between blend components through $\mathrm{C}=\mathrm{O} \ldots \mathrm{H}-\mathrm{C}-\mathrm{Cl}$ interactions increases with increase in PMMA concentration, but concluded the blends to be miscible in low PMMA concentration only.

From this review on PMMA/PVC blend systems, we observe that there seems to be some contradiction between our results and literature reports. We carefully analyzed the literature data and found some interesting factors. It has been convincingly said that miscibility in PMMA/PVC system is due to the hydrogen bonding type of interaction between the PMMA carbonyl group and $\alpha$-hydrogen of PVC. However, it has also been said that this bonding is not so strong that it alone can drive the system to miscibility. In such a situation, along with the intermolecular interactions, many other factors such as molecular weights and free volumes of the homopolymers, solvent used for blending, temperature conditions for preparation play a role in producing miscibility. In the backdrop of these aspects, we were able to notice a large difference in the molecular weights of the PMMA and PVC used in earlier studies and those used in the present study. In all most all earlier cases, the molecular weight of PMMA was observed to be greater than $100,000 \mathrm{~g} / \mathrm{mol}$ and PVC greater than $75,000 \mathrm{~g} / \mathrm{mol}$ respectively. In the present study, we have used PMMA and PVC with the molecular weights $15,000 \mathrm{~g} / \mathrm{mol}$ and 43,000 $\mathrm{g} / \mathrm{mol}$, respectively. This large difference in molecular weights of component polymers certainly seems to have its effect on the miscibility of the system. To cite one example to this end, Zacharius et al. [46] report that in case of PS/Poly(o-chlorostyrene) blends, a decrease in molecular weight of PS from 30,400 to $26,700 \mathrm{~g} / \mathrm{mol}$ turns a partially miscible system into miscible system.

In the light of this evidence, let us look at PMMA/PVC system. PMMA has two side groups, namely, methyl $\left(-\mathrm{CH}_{3}\right)$ and ester $\left(-\mathrm{COOCH}_{3}\right)$ groups, which make PMMA monomer relatively bulky compared to PVC. These groups in PMMA do not allow easy movement of the main chain as compared with PVC. From this aspect, we can consider PMMA to be bulky and rigid in relation to PVC. The chain entanglements in PMMA start from a molecular weight of $27,500 \mathrm{~g} / \mathrm{mol}$, which is referred as entanglement molecular weight of PMMA. Most of the PMMA samples used in the previous studies (literature data), in which the miscibility was observed in low PMMA concentrations, had PMMA molecular weight around 100,000 g/mol, which was well above the entanglement molecular weight $(27,500$ $\mathrm{g} / \mathrm{mol}$ ). Therefore, with the PMMA concentration increase, its morphology in the blend changed from dispersed phase to matrix and the entanglements between its chains grew rapidly. This restricted the dispersion of PVC in PMMA. By comparison, in the present study, we have used PMMA with a molecular weight of $15,000 \mathrm{~g} / \mathrm{mol}$, which is quite below its entanglement molecular weight. According to Wolf and Schnell's theory, reduction in entanglement density results in $\alpha$ negative. The higher negative $\alpha$ is supportive of this. Besides this, PMMA has larger free volume cavities and more free volume fraction than the PVC. Monomer molecular volumes of PMMA and PVC were calculated to be 87.9 $\AA^{3}$ and $47.5 \AA^{3}$, respectively. From these data, we can observe that PMMA free volume cavity $\left(115.6 \AA^{3}\right)$ can easily accommodate the PVC, but PVC free volume cavity $\left(72 \AA^{3}\right.$ ) can in no way accommodate the PMMA. This concept also enables us to interpret the fine and easy dispersion of PVC in PMMA is a real possibility, but not the other way round.

PVC/PS Blends. The second system we studied is PVC/ PS. PS does not possess any polar groups and forms an immiscible blend with PVC. Data presented in Fig. 6a and $b$ gives the variation of $V_{f}$ and $F_{v R}$ as function of PS concentration in the blend. Of the two homopolymers, PS has bigger free volume cavities $\left(98.2 \AA^{3}\right)$ and more free volume fraction $(31.6 \%)$ than the PVC $\left(72.2 \AA^{3}, 4.4 \%\right)$. Therefore, addition of PS into PVC obviously increases the average free volume cavity size and the free volume fraction. From Fig. 6a, we notice that free volume hole size increases rapidly with the addition of PS up to $20 \mathrm{wt} \%$. For $30 \mathrm{wt} \%$, it reaches a value almost equal to pure PS value and beyond $30 \mathrm{wt} \%$, it remains almost constant. This increase in free volume size up to $30 \%$ PS can be explained as follows. The PVC/PS blends are immiscible throughout the composition range and hence phase separated systems. The change in free volume size is from PVC value to PS value. It is well known that in polymers containing halogens like chlorine, fluorine, etc., the o-Ps formation probability is less since the halogens are inhibitors of o-Ps formation [39, 47, 48] hence smaller value of $\mathrm{I}_{3}$. As PS is added to PVC, more of 


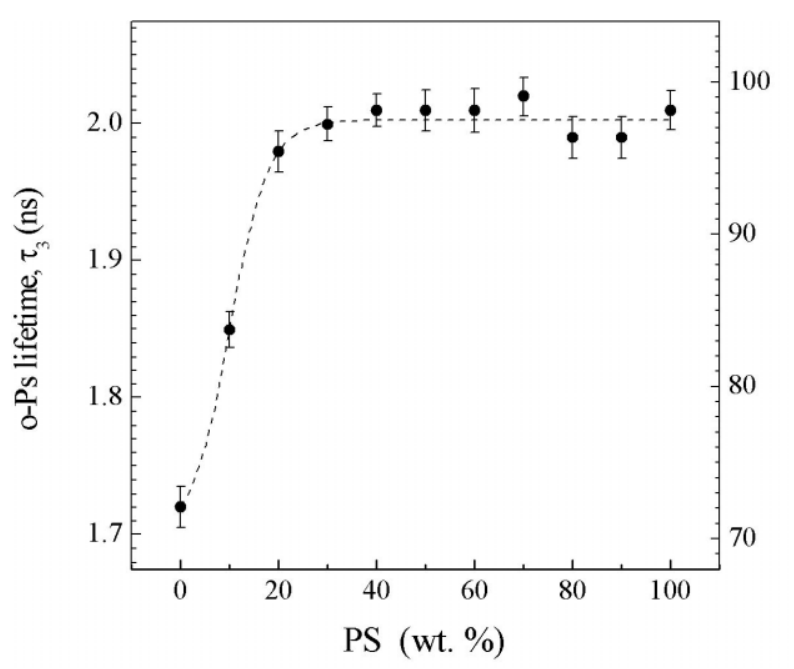

(a)

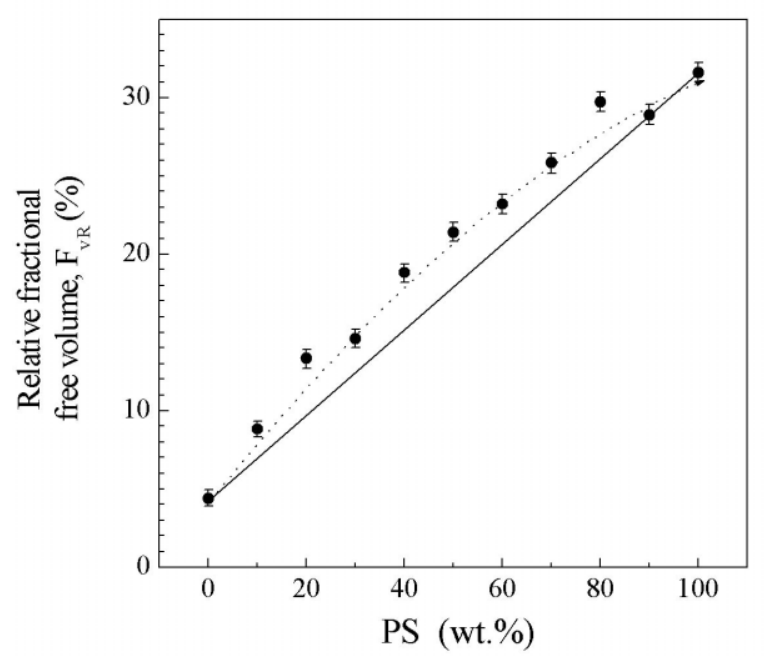

(b)

FIG. 6. (a) Plot of free volume hole size, $V_{f}$, as function of PS concentration in the blend. The solid line represents the linear additive relation and dotted line is to guide the eye. (b) Plot of relative fractional free volume, $\mathrm{F}_{\mathrm{vR}}$, as function of PS concentration in the blend. The solid line represents the linear additive relation and dotted line is to guide the eye.

positrons form o-Ps in PS than in PVC and 30\% of PS in PVC makes PS rich phase as far as o-Ps formation is concerned. Therefore, free volume size increases up to $30 \%$ of PS, beyond which for o-Ps seems to annihilate mainly in PS phase. The relative fractional free volume $\mathrm{F}_{\mathrm{vR}}$ (Fig. 6b) shows positive deviation throughout the concentration range. The free volume interaction parameter $\beta$ (Fig. 7) is also positive. On the basis of these, we can conclude that PVC/PS system as incompatible and immiscible.

For comparison purpose and also out of curiosity, we have performed calculations identical to PMMA/PVC system. The geometric factor $\gamma$ and hydrodynamic interaction parameter $\alpha$ have been evaluated and are plotted in Fig. 8a and $b$. It is interesting to observe that the hydrodynamic interaction parameter $\alpha$ is very small at all concentrations with a maximum value of -1.05 at $90 \mathrm{wt} \%$ of PS. As we have explained earlier, $\alpha$ measures the excess friction gen-

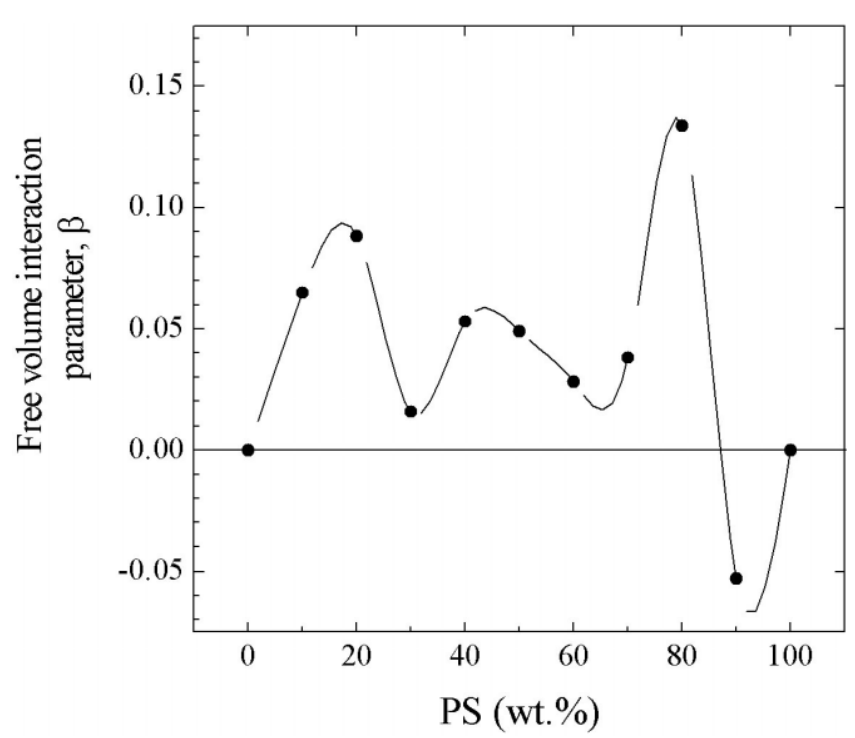

FIG. 7. Plot of the free volume interaction parameter $\beta$ as function of PS concentration in the blend. The dotted is to guide eye.

erated in system due to interaction between the constituent polymers; in case of immiscible blends, the possibility of contacts between the chains of component polymers is less because of the formation of domains of polymer 1 in the matrix of polymer 2 and vice versa. Therefore, the value of $\alpha$ is small in such systems, suggesting the system to be immiscible. The $\gamma$ parameter is also positive throughout the concentration range unlike its value that was negative in PMMA/PVC system.

\section{CONCLUSIONS}

Two blends, namely, PMMA/PVC and PVC/PS, have been studied using DSC, IR, and PLTs. In case of PMMA/ PVC, DSC measurements were inadequate to reveal convincingly whether the blends were miscible or not, since the $T_{\mathrm{g}} \mathrm{s}$ of pure polymers were not well separated. FTIR results indicated the shift in carbonyl band of PMMA in blends, suggesting the interactions between carbonyl group of PMMA and the $\alpha$-hydrogen of PVC. The behavior of free volume parameters, namely, average free volume hole size $\mathrm{V}_{\mathrm{f}}$, relative fractional free volume $\mathrm{F}_{\mathrm{vR}}$, and free volume interaction parameter $\beta$, suggest that the blends were miscible in low PVC concentrations. The variation of hydrodynamic interaction parameter $\alpha$ provided a way to predict the miscibility level to be high at $10 \%$ of PVC. The contradiction observed between our results and the earlier works is satisfactorily explained. This discrepancy was ascribed to the large differences in molecular weights of the homopolymers used in preparing the blends. This showed that in the absence of strong intermolecular interactions molecular weights and entanglement density of the polymers seems to play a major role in generating the miscibility.

PVC/PS blends were observed to be completely immiscible by DSC, as evidenced by the appearance of two glass transi- 


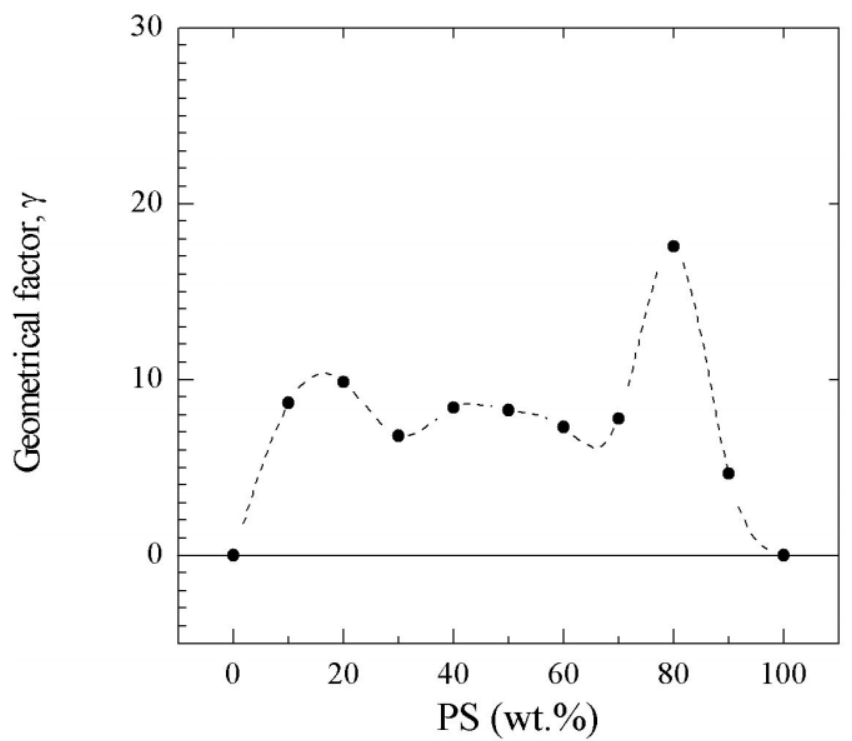

(a)

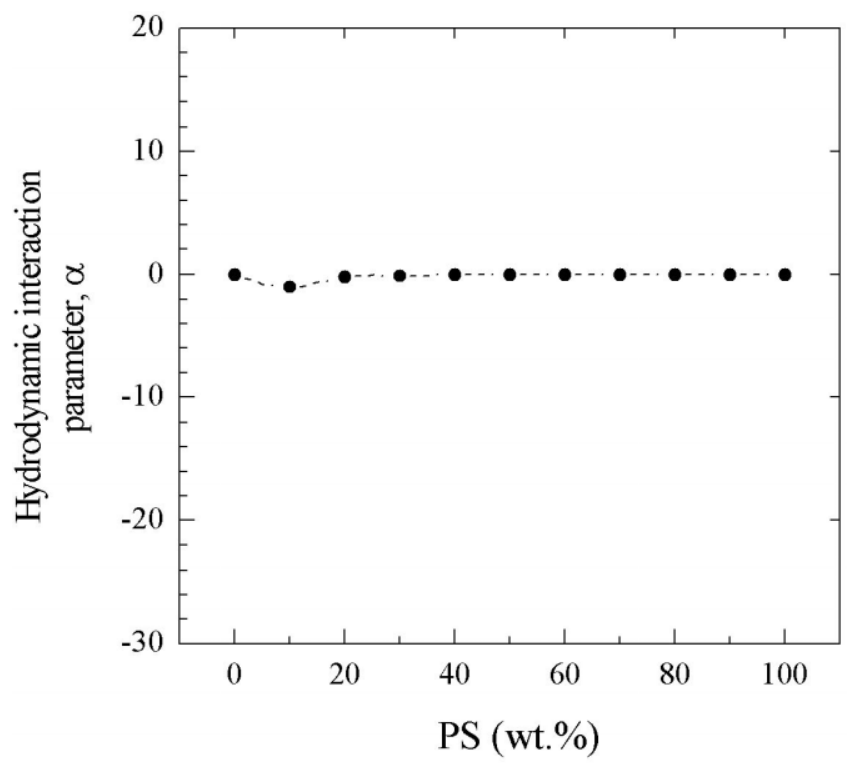

(b)

FIG. 8. (a) Plot of the geometrical parameter $\gamma$ as function of PS concentration in the blend. The dotted line is to guide eye. (b) Plot of hydrodynamic interaction parameter $\alpha$ as function of PS concentration in the blend. The dotted line is to guide eye.

tions in blends. The free volume parameters $\left(\mathrm{V}_{\mathrm{f}}, \mathrm{F}_{\mathrm{vR}}\right.$, and $\left.\beta\right)$ also clearly indicate the blends as immiscible. Hydrodynamic interaction parameter $\alpha$ for this system has small negative values, further suggesting the incompatibility. The present study is the first attempt to experimentally determine the $\gamma$ and $\alpha$ parameters of Wolf and Schnell theory based on free volume data instead of viscosity and suggest this theory could be used for polymer blends in solid phase as well.

\section{ACKNOWLEDGMENTS}

The authors thank the anonymous referees for their critical reading of the paper and fruitful suggestions, which in our belief has improved the presentation further. One of the authors (G. N. K) thank the Department of Science and Technology, Government of India, for providing him a Junior Research fellowship vide sanction No: SP/S2/M-18/ 2000 to carry out this research work.

\section{REFERENCES}

1. M.J. Folkes and P.S. Hope, Polymer Blends and Alloys, Chapman and Hall, London (1993).

2. L.A. Utracki, Text Book of Polymer Alloys and Blends (Thermodynamics and Rheology), Hanser, New York (1990).

3. O. Olabisi, L. Robeson, and M.T. Shaw, Polymer-Polymer Miscibility, Academic Press, New York (1979).

4. S.Y. Kwak, S.H. Kim, and T. Suzuki, Polymer, 45, 8153 (2004).

5. J. Liu, Y.C. Jean, and H. Yang, Macromolecules, 28, 5774 (1995).

6. P.J. Rouse, J. Chem. Phys., 21, 1272 (1953).

7. B. Zimm, J. Chem. Phys., 24, 279 (1956).

8. M. Schnell and B.A. Wolf, Polymer, 42, 8599 (2001).

9. J.W. Schurer, A. de Boer, and G. Challa, Polymer, 16, 201 (1975).

10. J. Vanderschueren, A. Janssens, M. Ladang, and J. Niezette, Polymer, 23, 395 (1982).

11. W.F. Lee and C.C. Lai, J. Appl. Polym. Sci., 66, 761 (1997).

12. J.F. Parmer, L.C. Dickinson, J.C.W. Chien, and R.S. Porter, Macromolecules, 22, 1078 (1989).

13. M.E. Fowler, J.W. Barlow, and D.R. Paul, Polymer, 28, 1177 (1987).

14. J.L.G. Pfennig, H. Keskkula, J.W. Barlow, and D.R. Paul, Macromolecules, 18, 1937 (1985).

15. H. Feng, Z. Feng, and C. Ye, Polym. J., 28, 661 (1996).

16. G. Shariff, P.M. Sathyanarayana, M.C. Thimmegowda, M.B. Ashalatha, R. Ramani, and C. Ranganathaiah, Polym. Degrad. Stab., 76, 265 (2002).

17. M.C. Thimmegowda, H.B. Ravikumar, and C. Ranganathaiah, J. Appl. Polym. Sci., 92, 355 (2004).

18. G. Dlubek, V. Bondarenko, Y. Al-Qaradawi, D. Kilburn, and R. Krause-Rehberg, Macromol. Chem. Phys., 205, 512 (2004).

19. H.B. Ravikumar, C. Ranganathaiah, G.N. Kumaraswamy, and S. Thomas, Polymer, 46, 2372 (2005).

20. R.F. Boyer, Polym. Eng. Sci., 8, 161 (1968).

21. J.P. Mercier, J.J. Aklonis, M. Litt, and A.V. Tobolsky, J. Appl. Polym. Sci., 9, 447 (1965).

22. R.R. Light and R.W. Seymour, Polym. Eng. Sci., 22, 857 (1982).

23. R.A. Bubeck, P.B. Smith, and S.E. Bales, Order in the Amorphous State of the Polymers, R.L. Miller and J.K. Ricke, editors, Plenum, New York, 347 (1987).

24. A.J. Hill, M.D. Zipper, M.R. Tant, G.M. Stack, T.C. Jordan, and A.R. Schultz, J. Phys. Condens. Matter., 8, 3811 (1996).

25. Y.C. Jean, Microchem. J., 42, 72 (1990).

26. P. Kirkegaard, N.J. Pedersen, and M. Eldrup, Riso Nat. Lab. 
Reports, RISO National Laboratory, Denmark, M-2740, (1989).

27. E.J. Vorenkamp and G. Challa, Polymer, 29, 86 (1988).

28. E. Fekete, E. Foldes, and B. Pukanszky, Eur. Polym. J., 41, 727 (2005).

29. D.F. Varnell and M.M. Coleman, Polymer, 22, 1324 (1981).

30. H. Nakanishi, Y.C. Jean, E.G. Smith, and T.C. Sandreczki, J. Polym. Sci. Part B: Polym. Phys., 27, 1419 (1989).

31. H. Nakanishi, S.J. Wang, and Y.C. Jean, Proceedings of International Symposium on Positron Annihilation Studies in Fluids, S.C. Sharma, editor, World Scientific, Singapore, 292 (1988).

32. S.J. Tao, J .Chem. Phys., 56, 5499 (1972).

33. M. Eldrup, D. Lightbody, and J.N. Sherwood, Chem. Phys., 63, 51 (1981).

34. F.N. Kelley and F. Bueche, J. Polym. Sci., 50, 549 (1961).

35. J.A. Campbell, A.A. Goodwin, M.S. Ardi, and G.P. Simon, Macromol. Symp., 118, 383 (1997).

36. S.J. Wu, J. Polym. Sci. Part B: Polym. Phys., 25, 2511 (1987).

37. J.A. Merrigan, J.H. Green, and S.J. Tao, "Techniques of Chemistry," in Physical Methods of Chemistry, Vol. 1, III D, A. Weissberger and B.W. Rossiter editors, Wiley, New York, 509 (1972).
38. A. Baranowski, M. Dêbowska, K. Jerie, G. Mirkiewicz, J. Rudzinska-Girulska, and R.T. Sikorski, J. Phys., IV, 225 (1993).

39. G. Dlubek, C. Taesler, G. Pompe, J. Pionteck, K. Petters, F. Redmann, and R. Krause-Rehberg, J. Appl. Polym. Sci., 84, 654 (2002).

40. M.L. Williams, R.F. Landel, and J.D. Ferry, J. Am. Chem. Soc., 77, 3701 (1955).

41. M. Kapnistos, A. Hinrichs, D. Vlassopoulos, S.H. Anastasiadis, A. Stammer, and B.A. Wolf, Macromolecules, 29, 7155 (1996).

42. A. Bondi, J. Phys. Chem., 6, 4418 (1964).

43. K. Venkateswaran, K.L. Cheng, and Y.C. Jean, J. Phys. Chem., 88, 2465 (1984).

44. P. Perrin, R.E. Prud'homme, Polymer, 32, 1468 (1991).

45. A.V. Rajulu, R. L. Reddy, S.M. Raghavendra, and S.A.K. Ahmed, Eur. Polym. J., 35, 1183 (1999).

46. S.L. Zacharius, G. ten Brinke, W.J. MacKnight, and F.E. Karasz, Macromolecules, 16, 381 (1983).

47. C.L.Wang, Y. Kobayashi, W. Zheng, C. Zhang, Y. Nagai, and M. Hasegawa, Phys. Rev. B, 63, 064204 (2001).

48. P. Ramachandra, R. Ramani, G. Ramgopal, G. Shariff, M.C. Thimmegowda, P.M. Sathyanarayana, C. Ranganathaiah, Polymer, 40, 5961 (1999). 